coding small RNAs, ranging from 18 to 25 nucleotides in length. MiRNAs are essential in regulating gene expression, cell development, differentiation and function. Dysregulation in miRNAs expression may contribute to the development of autoimmunity. However, a given miRNA may have hundreds of different mRNA targets and a target might be regulated by multiple miRNAs, thus the characterisation of dysregulated miRNA expression profiles could give a better insight into the development of immunological disturbances in autoimmune diseases.

Objectives: The aim of our study was to examine the changes in miRNA expression profiles in patients with primary Sjögren's syndrome (pSS) and systemic lupus erythematosus (SLE).

Methods: Eight pSS patients, 8 SLE patients and 7 healthy control subjects were enrolled in the investigation. MiRNAs were isolated from peripheral blood mononuclear cells, and expression patterns were determined with Illumina next-generation sequencing technology. Since the immunopathogenesis of pSS and SLE encompasses pronounced B cell hyperactivity along with specific autoantibody production, we paid a special attention on the association between miRNA expression levels and altered peripheral B cell distribution.

Results: In SLE patients 135, while in pSS patients 26 miRNAs showed altered expression. Interestingly, the 25 miRNAs including miR-146a, miR-16 and miR21 , which were over-expressed in pSS patients, were found to be elevated in SLE group, as well. On the contrary, we observed the down-regulation of miR-150-5p, which is a novel and unique finding in pSS. Levels of several miRNAs over-expressed in SLE, were not changed in pSS, such as miR-148a3p, miR-152, miR-155, miR-223, miR-224, miR-326 and miR-342. Expression levels of miR-223-5p, miR-150-5p, miR-155-5p and miR-342-3p, which miRNAs are potentially linked to B cell functions, showed associations with the B cell proportions within peripheral blood mononuclear cells.

Conclusions: The observed differences in miRNA expression profiles and the better understanding of immune regulatory mechanisms of miRNAs may help to elucidate the pathogenesis of pSS and SLE.

Acknowledgements: This work was supported by the ÚNKP-16-4-III New National Excellence Program of the Ministry of Human Capacities.

Disclosure of Interest: None declared

DOI: 10.1136/annrheumdis-2017-eular.3205

\section{AB0138 INTERFERON-GAMMA CHALLENGE OF PBMC FROM PATIENTS WITH LUPUS NEPHRITIS IN REMISSION DECREASES SUPPRESSOR OF CYTOKINE SIGNALING 1 (SOCS1) AND REGULATORY T CELLS (TREGS) AND PROMOTES IMMUNE ACTIVATION}

G. Gibor ${ }^{1}$, U. Arad ${ }^{1}$, J. Wallman ${ }^{1}$, J. Ablin ${ }^{1}$, V. Aloush ${ }^{1}$, I. Kaufman ${ }^{1}$, S. Jacky ${ }^{2}$, D. Caspi i ${ }^{1}$, O. Elkayam ${ }^{1}$, D. Paran ${ }^{1}$, A. Sharabi ${ }^{3} .{ }^{1}$ Rheumatology; ${ }^{2}$ Tel Aviv Medical Center, Tel Aviv, Israel; ${ }^{3}$ Harvard BIDMC, Boston MA, United States

Background: Interferon-gamma (IFN- $\gamma$ ) plays an important role in the development of lupus nephritis (LN). Regulation of IFN- $\gamma$ signaling that occurs in disease remission and in active $L N$ is herein addressed.

Objectives: To study the impact of IFN- $\gamma$ on PBMC obtained from patients with LN in remission as compared to active LN.

Methods: Sixteen patients fulfilling the ACR classification criteria for systemic lupus erythematosus were recruited. All patients had a history of $L N$ of whom 10 were in remission (as defined by EULAR criteria) and 6 had active LN (as defined by SLEDAI-2K or BILAG). Healthy subjects $(n=10)$ were included as a control group. Sera and PBMC were obtained from each individual. Flow cytometry, western blots and real time RT-PCR were used in processing and detection of cell subtypes, protein and mRNA levels. Recombinant human IFN- $\gamma($ rhIFN- $\gamma$ ) and anti-IFN- $\gamma$ neutralizing antibody were used in vitro. Mann-Whitney and student t-tests were used for statistical analysis.

Results: In active LN there was a significant 2-fold increase in $\mathrm{CD} 4^{+} \mathrm{CD} 69^{+}$ activated $\mathrm{T}$ cells as compared to healthy subjects and patients in remission. Reactivity to interferon-gamma receptor was determined by the phosphorylation of its predominant transcription factor, signal transducer and activator of transcription 1 (STAT1) in the cells that were incubated with rhIFN- $\gamma$ or with media alone. In active LN, 3- and 6-fold increase in pSTAT1 occurred with rhIFN- $\gamma$ incubation during $24 \mathrm{~h}$ and $48 \mathrm{~h}$, respectively, and healthy subjects responded likewise. In patients in remission, pSTAT1 increase was even higher (by 8- and 10-fold at 24h and $48 \mathrm{~h}$, respectively). After $24 \mathrm{~h}$ incubation with rhIFN-g all groups had elevated (mRNA) expression of SOCS1, but at $48 \mathrm{~h}$, it significantly decreased in healthy subjects and in patients in remission by $34 \%$ and $50 \%$, respectively. Further, at $24 \mathrm{~h}$ the frequency of $\mathrm{CD}^{+} \mathrm{CD} 127^{\text {low }} \mathrm{FoxP}^{+}$regulatory $\mathrm{T}$ cells (e.g. Tregs) increased by $27-30 \%$ in active LN and in healthy subjects, and in remission it was minimally changed. At $48 \mathrm{~h}$, the frequency of Tregs significantly decreased in healthy subjects (to baseline levels before rhIFN- $\gamma$ challenge) and in patients in remission (by $24 \%, p=0.003$ ). A challenge of PBMC from $L N$ patients in remission with sera (at $1 \%$ concentration) derived from patients with active LN resulted in 7.5-fold increase in PSTAT1 expression and 20-30\% decrease in Tregs, however, combination of sera and anti-IFN-g neutralizing antibody resulted in 5-fold decrease in PSTAT1 expression and significantly diminished the decrease in Tregs.

Conclusions: In LN in remission a challenge with IFN- $\gamma$ could lead to immune activation and a risk of flare-up, as it results in a decrease in both SOCS1 and
Tregs and a robust STAT1 phosphorylation. In active LN, STAT1 phosphorylation is less diminishing, as both SOCS1 and Tregs are saturated, which could affect their suppressive effectiveness.

Disclosure of Interest: None declared

DOI: 10.1136/annrheumdis-2017-eular.4281

\section{AB0139 ROLE OF MUCOSAL-ASSOCIATED INVARIANT T (MAIT) CELLS} IN A LUPUS MODEL

G. Murayama ${ }^{1,2}$, A. Chiba ${ }^{2}$, A. Nomura ${ }^{2}$, H. Amano ${ }^{1}$, K. Yamaji ${ }^{1}$, N. Tamura ${ }^{1}$, S. Miyake ${ }^{2} \cdot{ }^{1}$ Department of Internal Medicine and Rheumatology; ${ }^{2}$ Department of Immunology, Juntendo University School of Medicine, Tokyo, Japan

Background: Mucosal-associated invariant T (MAIT) cells are innate T cells that are restricted by MHC-related molecule-1 (MR1) and express a semi-invariant TCR $\alpha$ chain: V $\alpha 7.2-J \alpha 33$ in humans and $V_{\alpha} 19-J \alpha 33$ in mice. Previously, we have demonstrated that MAIT cells played a protective role against experimental autoimmune encephalomyelitis, an animal model of human multiple sclerosis. We found that MAIT cells are activated in patients with systemic lupus erythematosus (SLE) and that the activation state of MAIT cells correlated with SLE disease activity index (SLEDAI) score, suggesting their association in lupus pathology. Objectives: We set out to clarify functions of MAIT cells in a lupus model by using $\mathrm{F}_{\mathrm{C}} \gamma \mathrm{RIIB}^{-/-}$Yaa mice.

Methods: Fc $\gamma$ RIIB $^{-/}$Yaa mice were crossed to MR1 deficient mice lacking MAIT cells, and disease progression was compared between MR1 ${ }^{-/} \mathrm{Fc} \mathrm{RIIB}^{-1-}$ Yaa and MR1 ${ }^{+/+} \mathrm{Fc} \gamma \mathrm{RIIB}^{-1-}$ Yaa mice at 1-4 months of age. Serum anti-dsDNA antibody levels were measured and urinary microalbumin were evaluated. At the time of sacrifice, at 4 months of age, the severity of nephritis and dermatitis were assessed by histologically and IgG deposition in skin and glomeruli was measured.

Results: Survival rate was significantly reduced in $\mathrm{MR}^{-1} \mathrm{Fc} \gamma \mathrm{R} / \mathrm{ll} \mathrm{b}^{-/}$Yaa mice compared with MR1//+ FcyRllbb-/- Yaa mice. Anti-dsDNA antibody levels were remarkably higher in $\mathrm{MR}^{+/+}$than $\mathrm{MR} 1^{-/} \mathrm{Fc} \gamma \mathrm{R} / \mathrm{ll}{ }^{-/}$Yaa mice at 4 months of age. Even though Glomeruli were significantly enlarged both in $\mathrm{MR} 1^{+/+}$and $\mathrm{MR}^{-1-} \mathrm{Fc} \gamma \mathrm{RIIb}^{-1-}$ Yaa mice due to a marked cellular proliferation in glomeruli, the glomerulonephritis score tended to be lower in $\mathrm{MR}^{-/} \mathrm{Fc} \gamma \mathrm{Rllb}{ }^{-/}$Yaa mice compared with $\mathrm{MR}^{+/+} \mathrm{Fc} \gamma \mathrm{R} / \mathrm{ll} \mathrm{b}^{-1-}$ Yaa mice. A larger amount of IgG deposition was observed in mesangial area and along glomerular capillary walls in

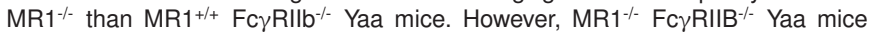
showed exacerbated inflammation in the skin lesions. There was a high degree of inflammatory cells infiltration into the skin and a significant worsening of dermatitis

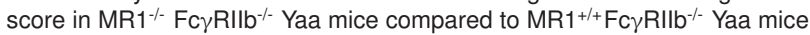
Conclusions: These data suggests that MAIT cells exhibit dual roles in lupus pathogenesis. MAIT cells enhance autoantibody production and the disease severity of nephritis, but have a suppressive effect on dermatitis. Further studies are under going to uncover the mechanisms by which MAIT cells are involved in each target tissues.

References:

[1] Croxford JL, et al. Invariant V(alpha)19i T cells regulate autoimmune inflammation. Nat Immunol. 2006; 7(9): 987-94.

[2] Chiba A, et al. Mucosal-associated invariant $T$ cells promote inflammation and exacerbate disease in murine models of arthritis. Arthritis Rheum. 2012; 64(1): 153-61.

[3] Kawano S, et al. Phenotype conversion from rheumatoid arthritis to systemic lupus erythematosus by introduction of Yaa mutation into Fc $\gamma$ RIIIB-deficient C57BL/6 mice. Eur J Immunol. 2013; 43(3): 770-8.

Disclosure of Interest: None declared

DOI: 10.1136/annrheumdis-2017-eular.3086

\section{AB0140 LUPUS-PRONE SLAM HAPLOTYPE EXERTS MONOCYTOSIS AND DEVELOPS SPECIFIC PHENOTYPE OF AUTOIMMUNE DISEASE INTRODUCED BY YAA MUTATION}

H. Amano ${ }^{1}$, K. Nishikawa ${ }^{2}$, Q. Lin ${ }^{3}$, S. Kawano ${ }^{1}$, K. Yamaji ${ }^{1}$, S. Hirose ${ }^{3}$, N. Tamura ${ }^{1} .{ }^{1}$ Department of Internal Medicine and Rheumatology; ${ }^{2}$ Department of Pathology, Juntendo University School of Medicine, Tokyo; ${ }^{3}$ Department of Biomedical Engineering, Toin Human Science and Technology Center, Toin University of Yokohama, Yokohama, Japan

Background: We previously obtained a 129-derive Fc $\gamma R$ RIIB-deficient C57BL/6 (B6) congenic strain of mice, which spontaneously developed severe rheumatoid arthritis (RA) ${ }^{1}$. The introduction of the Yaa (Y-linked autoimmune acceleration) mutation, which is a consequence of a translocation from the telomeric end of the $\mathrm{X}$ chromosome containing the TIr7 gene onto the $\mathrm{Y}$ chromosome, to the Fc $\gamma$ RIIIB-deficient B6 mice (B6.Fc $\gamma$ RIIB-/.. Yaa) developed lupus like nephritis but not $\mathrm{RA}^{2}$.

Objectives: By extensively backcrossing 129-based Fc $\gamma$ RIIIB-deficient mice to B6 mice, we established wildtype Fc $\gamma R$ RIIB and 129-derive autoimmune-prone SLAM haplotype $\left(S / a m^{129}\right)$. We examined the phenotype of Slam ${ }^{129}$ mice, and also $S^{\prime a m}{ }^{129}$. Yaa mice by introducing Yaa mutation to these mice.

Methods: We analyzed peripheral blood monocyte subset and also serum autoantibodies as well as immunohistopathological findings of kidneys and lungs. Results: Slam ${ }^{129}$ mice showed age-associated monocytosis with marked expan- 